\title{
EFECTO DE LA TEMPERATURA ESCROTAL SOBRE EL COMPORTAMIENTO SEXUAL Y LA CALIDAD DE SEMEN DE OVINOS PELIBUEY Y MERINO PRECOZ ALEMÁN
}

\author{
Yolanda Manco M.', Víctor Leyva V., José Camacho S. ${ }^{3}$ y Sergio Cueva M. ${ }^{4}$
}

\section{Abstract}

The effect of increased scrotal temperature on semen quality, sexual behavior and the recovery of normal semen production was studied using 3 Pelibuey $(\mathrm{OPb})$ and 3 German Merino (OMP) sheep. An insulated bag was applied to the testicles for a period of six days, during which the average ambient temperature and relative humidity were $23.2 \pm 1.1^{\circ} \mathrm{C}$ and $81 \pm 2 \%$. Using an artificial vagina, semen was collected twice weekly for two weeks prior to initiation and once weekly for nine weeks post-treatment.

: Sexual libido

of the rams was measured on the day of semen collection. During the six days of insulated scrotal bag treatment, scrotal $\left(\mathrm{T}^{\circ} \mathrm{E}\right)$, rectal $\left(\mathrm{T}^{\circ} \mathrm{R}\right)$ and skin $\left(\mathrm{T}^{\circ} \mathrm{P}\right)$ temperatures were recorded. Increases of $\mathrm{T}^{\circ} \mathrm{E}$ to $37.1 \pm 0.9^{\circ} \mathrm{C}$ in $\mathrm{OPb}$ and $38.2 \pm 0.7^{\circ} \mathrm{C}$ in $\mathrm{OMP}$ were registered which decreased $(\mathrm{P}<0.01)$ two to three weeks post-treatment. Evaluation of sperm concentration and motility revealed increased seminal $\mathrm{pH}$ and an elevated frequency of abnormal and dead spermatozoa, although no change in seminal volume or sexual libido occurred. Breed*week interaction was significant $(P<0.001)$ due to the greater susceptibility of $\mathrm{OMP}$ and the early recovery of normal testicular function in $\mathrm{OPb}$. The highly significant $(\mathrm{P}<0.0001)$ correlation of $T^{\circ} E$ with $T^{\circ} R(r=0.63)$ and $T^{\circ} P(r=0.65)$ suggests that the ability of $\mathrm{OPb}$ to compensate the heat effect may be due to the efficiency of the skin in dissipating heat, rather than a thermal regulation mechanism of the testicles.

Key words: Sheep, scrotal temperature, semen, libido.

\section{Restmen}

El efecto del incremento de la temperatura escrotal sobre la calidad de semen, comportamiento sexual y el inicio de regeneración de la producción normal de semen fue estudiado en tres ovinos Pelibuey (OPb) y tres Merino Precoz Alemán (OMP) mediante bolsas térmicas aplicadas a los testiculos durante seis días en un medio ambiente con temperatura promedio de $23.2 \pm 1.1^{\circ} \mathrm{C}$ y humedad relativa de $81 \pm 2 \%$. Dos colecciones de semen con vagina artificial se realizó cada semana durante una fase pre-experimental de dos semanas antes del tratamiento y una fase experimental de nueve semanas después del tratamiento y una colección semanal durante una fase post-experimental para precisar el periodo de regeneración seminal. Variación en tiempo que cada macho tomó para montar a la hembra en cada colección de semen fue expresado como variación del líbido sexual. Durante el tratamiento se registraron la temperatura escrotal $\left(\mathrm{T}^{\circ} \mathrm{E}\right)$, rectal $\left(\mathrm{T}^{\circ} \mathrm{R}\right) \mathrm{y}$ de la piel (ToP). El incremento de la $\mathrm{T}^{\circ} \mathrm{E}$ a $37.1 \pm 0.9^{\circ} \mathrm{C}$ en $\mathrm{OPb}$ y a $38.2 \pm 0.7^{\circ} \mathrm{C}$ en OMP, 203

\footnotetext{
'Práctica privada

${ }^{2}$ Laboratorio de Reproducción - FMV - UNMSM

${ }^{3}$ Departamento de Producción Animal - FMV - UNMSM

- Laboratorio de Fisiologia - FMV - UNMSM
} 
semanas después, disminuyó $(\mathrm{P}<0.01)$ la concentración y motilidad espermática y la circunferencia escrotal $(\mathrm{P}<0.01)$ y aumentó $(\mathrm{P}<0.01)$ el pH seminal y la frecuencia porcentual de espermatozoides anormales y muertos; sin embargo no hubo efecto en el volumen seminal y libido sexual. El efecto en estas alteraciones dependió de la interacción raza*semana $(\mathrm{P}<0.001)$ debido a una mayor susceptibilidad del OMP y a un inicio de regeneración más temprano en $\mathrm{OPb}$. En base a la alta correlación significativa $(\mathrm{P}<0.0001)$ de la $T^{\circ} \mathrm{E}$ con la $\mathrm{T}^{\circ} \mathrm{R}(\mathrm{r}=0.63)$ y la $\mathrm{T}^{\circ} \mathrm{P}(\mathrm{r}=0.65)$ se sugiere que la habilidad del $\mathrm{OPb}$ para compensar el efecto del calor, radicaría en la eficiencia de la cobertura de su piel para disipar el calor en lugar de un eficiente mecanismo termorregulador de los testículos.

Palabras clave: Ovino, temperatura escrotal, semen, líbido.

\section{Introducción}

Factores como cambios en fotoperíodo e incremento de la temperatura medioambiental (Moule y Waites 1963; Lincoln, 1992) pueden influir en la función reproductiva del carnero.

Es conocido también el efecto negativo del incremento de la temperatura sobre la espermatogénesis testicular debido a la aplicación local de calor a nivel testicular (Waites y Setchell, 1964; Hochereau-de Reviers et.al., 1993) y se estima que la respuesta del animal al efecto de la temperatura puede depender de la raza en relación al medio donde fue desarrollada.

Estudios en ovinos indican que la raza Pelibuey, de zonas tropicales, es resistente a temperaturas promedio de $30^{\circ} \mathrm{C}$, pero la presión osmótica del semen es susceptible a la humedad ambiental (Carmenate et.al., 1982). Sin embargo, se desconoce si el incremento de temperatura en un ambiente de mayor humedad, como en las zonas tropicales y costeras del país, compromete su performance reproductiva. Tampoco existen estudios similares, en nuestro medio, para Merino precoz Alemán, no obstante, reportes indican que esta raza se adapta bien a condiciones semiáridas (Degen y Benjamin, 1987).

En base a estas informaciones se planteó el presente trabajo con el objetivo de evaluar el efecto del incremento de la temperatura escrotal en un ambiente húmedo
(Lima) sobre el comportamiento sexual, calidad de semen y el periodo de regeneración de la producción normal de semen, en ovinos Pelibuey y Merino Precoz Alemán.

\section{Materiales y Mctotos}

\section{Lugar de estudio $y$ animales}

El presente estudio se llevó a cabo en verano (enero-marzo), en la Unidad de Producción de Ovinos de la Facultad de Medicina Veterinaria de la UNMSM, departamento de Lima, ubicado a una altitud de 72 msnm. La temperatura y humedad relativa ambiental promedio durante el periodo de estudio fueron $23.2 \pm 1.1^{\circ} \mathrm{C}$ y $81 \pm 2 \%$, respectivamente.

Después de evaluar la condición física y clínica corporal de los órganos genitales y el comportamiento sexual a un grupo de ovinos machos nacidos en la Unidad de producción, se seleccionaron al azar 6 carneros (3 Pelibuey y 3 Merino Precoz Alemán) de edades similares (2.5-3.5 años). Todos los carneros fueron previamente entrenados para el manejo y la colección de semen con vagina artificial y ubicados en un corral donde recibieron la misma ración alimenticia (chala y concentrado) con agua ad libitum. El peso corporal promedio al inicio y al final del experimento fue $54.33 \pm 12.1$ y $54.50 \pm$ $11.35 \mathrm{~kg}$, respectivamente. 


\section{Diseño experimental}

Los seis carneros seleccionados al azar fueron distribuidos de acuerdo al siguiente diseño experimental:

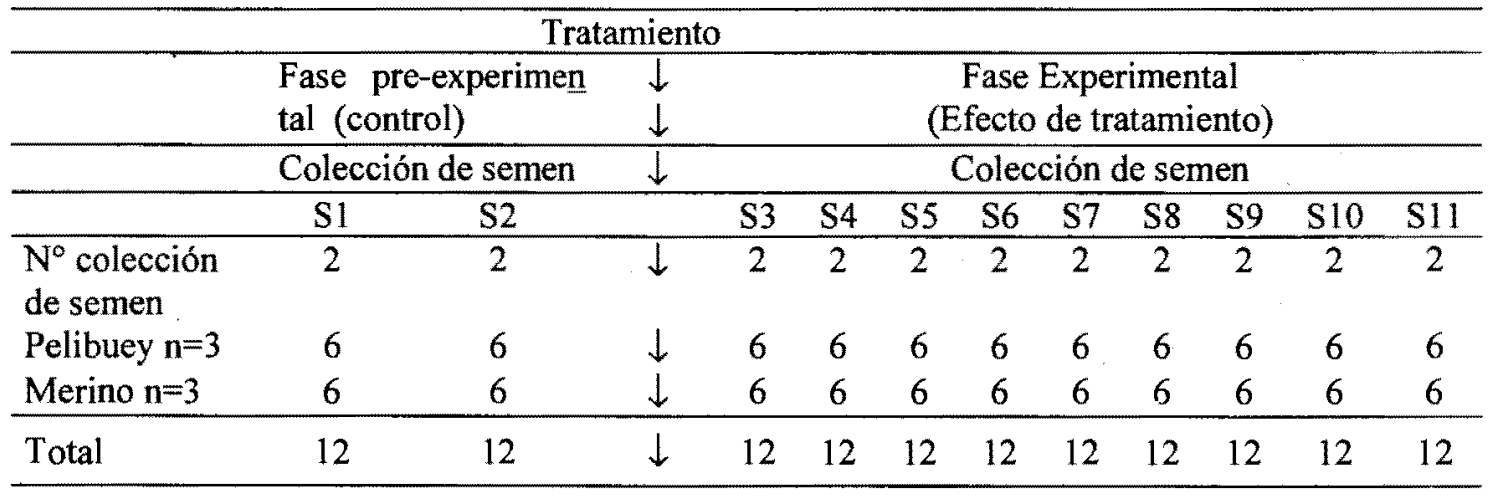

Se consideraron 2 fases, una pre-experimental que constituyó el control (sin tratamiento), y la fase experimental correspondió al efecto del tratamiento (aplicación de bolsas térmicas a testículos). La primera fase se realizó en las semanas 1 y 2 ( $\mathrm{S} 1$ y $\mathrm{S} 2$ ) y la segunda fase desde la semana 3 hasta la 11 (S3 a S11). Desde la S1 hasta la S11 se realizaron dos colecciones de semen por semana (lunes y jueves) en cada animal de cada raza.

Se consideró una tercera fase, postexperimental desde la semana 13 hasta la 15 a fin de precisar el periodo de regeneración; en esta fase se realizó una colección de semen por semana en cada animal.

\section{Método de tratamiento}

Para el incremento de la temperatura escrotal se utilizaron bolsas térmicas preparadas en el laboratorio con fibra nylon y polibrillo. Las bolsas térmicas fueron expuestas al calor del sol y su capacidad de absorción y retención de calor fueron medidos con termómetro cada hora entre las 12:00 y 15:00 horas; las bolsas que mostraron valores térmicos extremos del promedio fueron modificadas a fin de reducir la variabilidad. Así la temperatura promedio de las bolsas en las horas de mayor temperatura del medio ambiente (12:00 a 15:00 horas) fue de $41.25^{\circ} \pm$ $0.1^{\circ} \mathrm{C}$. Cuando estas bolsas fueron aplicadas a los testículos de los carneros (independientemente de las razas), la temperatura promedio escrotal máxima fue de $37.9^{\circ}$ entre las 12:00 y 13:00 horas. En los ovinos Pelibuey, se adicionó fibra de drylon alrededor de los testículos a fin de simular la presencia de lana, como ocurre en los ovinos Merino Precoz.

El inicio del tratamiento con bolsas escrotales fue al día siguiente (viernes) de la segunda colección de la semana 2 y terminó 2 días después de la primera colección de la semana 3. Durante el tratamiento se registraron cada hora, entre las 12:00 y 16:00 horas, las temperatura escrotal, rectal y de la piel (del flanco derecho) en todos los animales. Datos sobre humedad relativa y temperatura ambiental diaria durante el periodo de estudio (enero-marzo) fueron obtenidos del centro metereológico de la Universidad Agraria de la Molina, localizado aproximadamente a $8 \mathrm{~km}$. del área de estudio.

\section{Colección de semen}

Se utilizó la técnica de la vagina artificial descrita por Sorensen (1991). La colección de semen se realizó entre 8:00 y 11:00 horas en los respectivos días. El semen fue colectado en un tubo graduado e inmediatamente después de su obtención fue conservado a $35^{\circ}$ durante todo el proceso de su evaluación. 


\section{Evaluación del Comportamiento Sexual}

Antes de la colección de semen, en las 3 fases, se evaluó el comportamiento sexual de los carneros (cortejo, monta, erección y eyaculación) en presencia de las hembras. Para la evaluación se utilizó una escala del 0 al 2 , donde 0 representó ausencia del interés sexual; 1 , presencia del interés sexual pero con tiempo prolongado (igual o mayor a 3 minutos) para realizar la monta; y 2 , gran interés sexual con rápida monta.

\section{Método de Evaluación del Semen}

Las características fisicas del semen fueron evaluadas con técnicas descritas por Evans y Maxwell (1987). El volumen de eyaculado fue medido en la escala graduada del tubo colector y el pH se determinó con tiras indicadoras de pH (Merk, Spezialindikator $\mathrm{pH}$, con escala de 6.5 a 10.0 con incremento de 0.3 ).

Microscópicamente se evaluó la motilidad masal de una gota de semen fresco utilizando la escala del 0 al 5 descrita por Moule y Waites (1963). La motilidad progresiva (porcentaje de espermatozoides que se desplazan progresivamente en varios campos al azar) se evaluó usando semen diluido (una gota de semen en $3 \mathrm{ml}$ de dilutor). Como dilutor se utilizó glucosa al $0.55 \%$ y citrato al $2.8 \%$.

La concentración de espermatozoides ( $\mathrm{x} 10^{6} / \mathrm{ml}$.) fue determinada con la cámara de NewBauer (Sorensen, 1991).

Para la determinación del porcentaje de espermatozoides muertos y anormales, se hicieron frotices del semen de cada eyaculado inmediatamente después de la colección. Se utilizó la coloración nigrosina-eosina (Hancock, 1952) y en cada frotis se evaluaron diversos campos hasta contar en total 200 espermatozoides para cada variable.

\section{Análisis estadístico}

El efecto del tratamiento y de la raza $y$ de sus interacciones en las variables continuas (volumen, $\mathrm{pH}$ y concentración) fueron evaluados con el diseño de "parcelas divididas» con análisis de varianza (ANOVA) (SAS Institute, 1990), donde la sparcela mayor» lo constituyó la raza y la «parcela menor» semana de colección de semen.

El modelo matemático lineal fue:

$$
\begin{aligned}
& Y=\text { raza animal(raza) sem }
\end{aligned}
$$

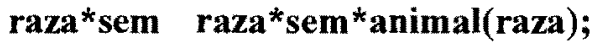

En este modelo se utilizó la variable nestada animal(raza) para evaluar el efecto de la parcela mayor raza; y la interacción raza*sem*animal(raza) para evaluar en la parcela menor el efecto de semana de colección y el efecto de raza en la interacción sem*raza. Sólo en caso que la interacción fue significativa se utilizó el «promedio del cuadrado mínimo» del programa GLM, para determinar en que semana después del tratamiento hubo influencia significativa de la raza, confrontándolo con las semanas control.

Para las variables discontinuas (motilidad masal y progresiva, porcentajes de espermatozoides anormales y muertos, y comportamiento sexual) se utilizó la tabla de contingencia $\langle\mathrm{r} \times \mathrm{c}$ ) del chi cuadrado (Steel y Torrie, 1960) donde «r» lo constituyó las semanas de colección de semen y «c» la raza para evaluar la existencia de interacción entre las variables. En caso de interacción significativa, la detección de la semana después del tratamiento donde la raza influyó significativamente fue determinada por chi cuadrado comparando cada semana después del tratamiento vs. semanas control en cada raza. 


\section{Resultatios}

\section{Concentración espermática}

No hubo efecto de raza, pero si hubo efecto significativo $(\mathrm{P}<0.0001)$ de semana entre las semanas de colección antes del tratamiento y cada semana después del tratamiento, observándose esta diferencia a partir de la semana 5 y continuó más halla de la semana 11. La interacción raza*sem tuvo tendencia significativa $(\mathrm{P}<0.06)$, en donde dicha tendencia se observó a partir de la semana 6 y desapareció (inicio de regeneración) en la semana 8 en la raza Pelibuey mientras que en Merino Precoz esto ocurrió a partir de la semana 5 y desapareció (inicio de regeneración) en la semana 15 (Figura 1).

Las anormalidades espermáticas más frecuentes, en orden descendente, fueron: Cabeza desprendida, cola doblada, pieza intermedia deshilachada y acrosoma anormal.

\section{Porcentaje de espermatozoides muertos}

La frecuencia de espermatozoides muertos empezó a incrementarse a partir de la semana 4 en ambas razas; sin embargo el incremento en cada semana dependió de la raza, ya que la interacción raza* sem fue significativa $(\mathrm{P}<0.005)$ y se manifestó con un inicio de regeneración más temprano en el ovino Pelibuey (semana 8), mientras que en el Merino Precoz ocurrió en la semana 13 (Figura 1).

\section{Motilidad masal y progresiva}

La interacción raza* ${ }^{*}$ sem fue significativa $(\mathrm{P}<0.005)$ para motilidad masal y progresiva, ya que empezaron a disminuir a partir de la semana 4 en ambas razas; sin embargo esta disminución significativa desapareció en el ovino Pelibuey en la semana 8 , mientras que en Merino Precoz esto ocurrió en la semana 14. (Figura 2).

\section{pH y volumen seminal.}

En pH seminal no hubo efecto de raza; sin embargo hubo efecto significativo $(P<0.0001)$ de semana entre las semanas de colección antes del tratamiento y cada semana después del tratamiento, observándose esta diferencia a partir de la semana 5 hasta la 10. Hubo tendencia a la significancia $(\mathrm{P}<0.07)$ de la interacción raza*sem, en donde la significancia desapareció a partir de la semana 8 en la raza Pelibuey mientras que en Merino Precoz ocurrió a partir de la semana 11 (Figura 2).

No hubo efecto significativo de raza y de interacción raza*sem en el volumen seminal; sin embargo, hubo tendencia a la significancia $(\mathrm{P}<0.06)$ entre semanas de colección antes del tratamiento y la semana 5 $(\mathrm{P}<0.06)$.

\section{Comportamiento Sexual}

No hubo interacción significativa raza*sem ni efecto simple de raza y semana de colección en el comportamiento sexual de los ovinos Pelibuey y Merino Precoz.

Temperatura Escrotal $\left(\mathrm{T}^{\circ} \mathrm{E}\right)$, Temperatura Rectal $\left(T^{\circ} R\right)$ y temperatura de la Piel ( $\left.\mathbf{T}^{\circ} \mathbf{P}\right)$

La máxima $\mathrm{T}^{\circ} \mathrm{E}$ (registrada entre las 12:00 y 16:00 horas) obtenida con las bolsas térmicas «in situ» en los testículos de los carneros fue diferente $(\mathrm{P}<0.05)$ entre ovino Pelibuey $\left(37.1^{\circ} \pm 0.9^{\circ} \mathrm{C}\right)$ y Merino Precoz $\left(38.2 \pm 0.7^{\circ}\right)$. La máxima $\mathrm{T}^{\circ} \mathrm{E}$ coincidió con las temperaturas ambientales máximas (rango de 26.1 a $28.2^{\circ} \mathrm{C}$ ) alcanzadas.

También hubo diferencia significativa $(\mathrm{P}<0.05)$ de la $\mathrm{T}^{\circ} \mathrm{R}$ y $\mathrm{T}^{\circ} \mathrm{P}$ durante el tratamiento entre las razas Pelibuey $\left(\mathrm{T}^{\circ} \mathrm{R}\right.$ : $39.2 \pm 0.6^{\circ} \mathrm{C}$ $\mathrm{T}^{\circ} \mathrm{P}: 39.8 \pm 1.0^{\circ} \mathrm{C}$ ) y Merino Pre$\operatorname{coz}\left(\mathrm{T}^{\circ} \mathrm{R}: 39.9 \pm 0.6^{\circ} \mathrm{C}\right.$ y $\mathrm{T}^{\circ} \mathrm{P}: 41.5 \pm 1.3^{\circ} \mathrm{C}$ ).

Hubo correlación significativa $(\mathrm{P}<0.0001)$ de $T^{\circ} E$ con $T^{\circ} R(r=0.63)$ y con $T^{\circ} P(r=0.65)$.

Durante los días del tratamiento la temperatura ambiental promedio fue $21.7 \pm 0.7^{\circ} \mathrm{C}$ y la humedad relativa promedio $80 \pm 2 \%$, alcanzando valores máximos de $28.2^{\circ} \mathrm{C}$ y $97 \%$, respectivamente. 

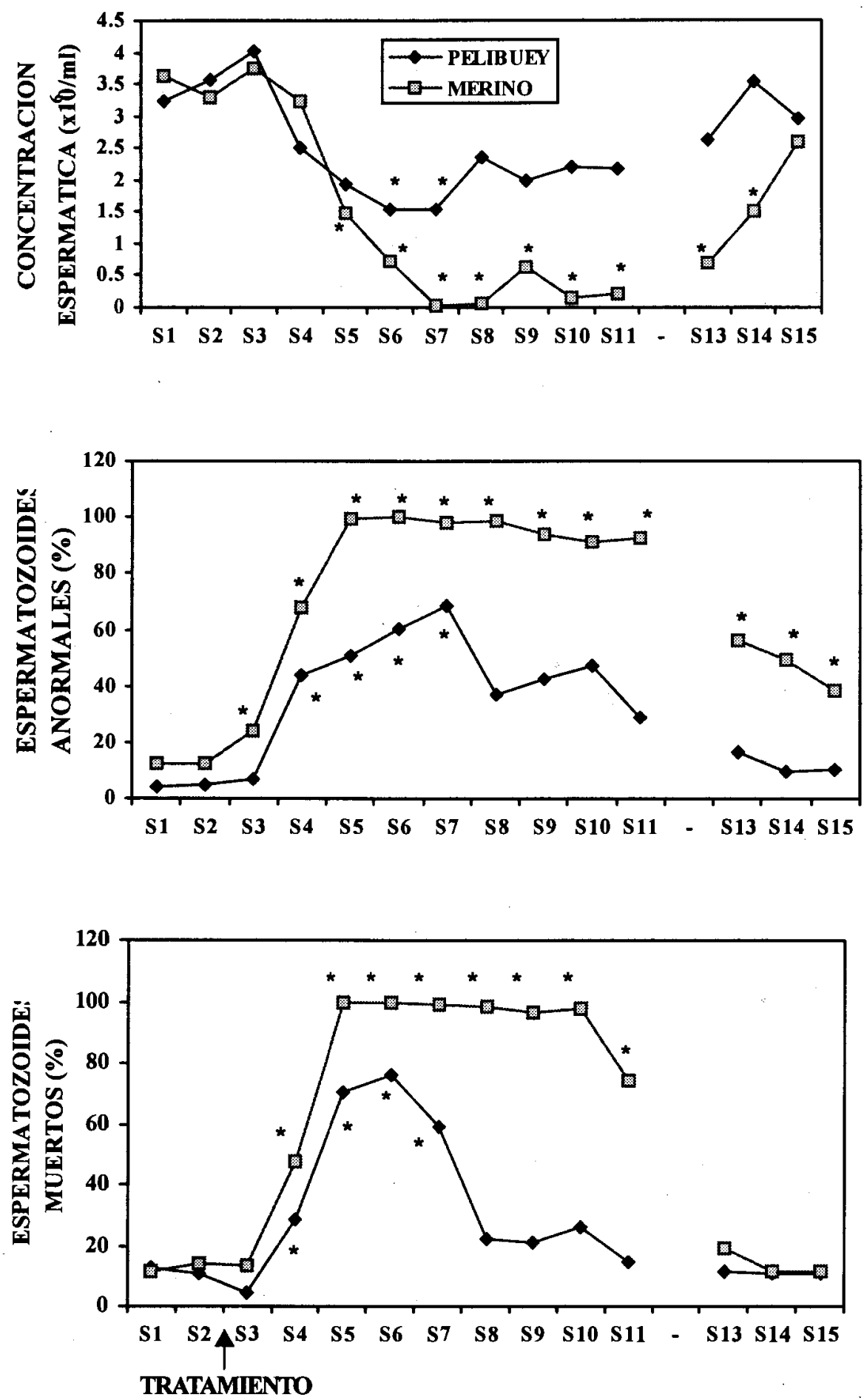

\section{SEMANAS DE COLECCIÓN DE SEMEN}

Figura 1. Efecto del incremento de la temperatura escrotal sobre la concentración espermática y porcentajes de espermatozoides anormales y muertos en ovinos Pelibuey y Merino Precoz durante las fases pre-experimental o control (S1 y S2), experimental (S3 a S11) y post-experimental (S13 a S15). Los * indican la diferencia significativa de los promedios de las semanas post-tratamiento respecto al promedio de las semanas control en cada raza. 

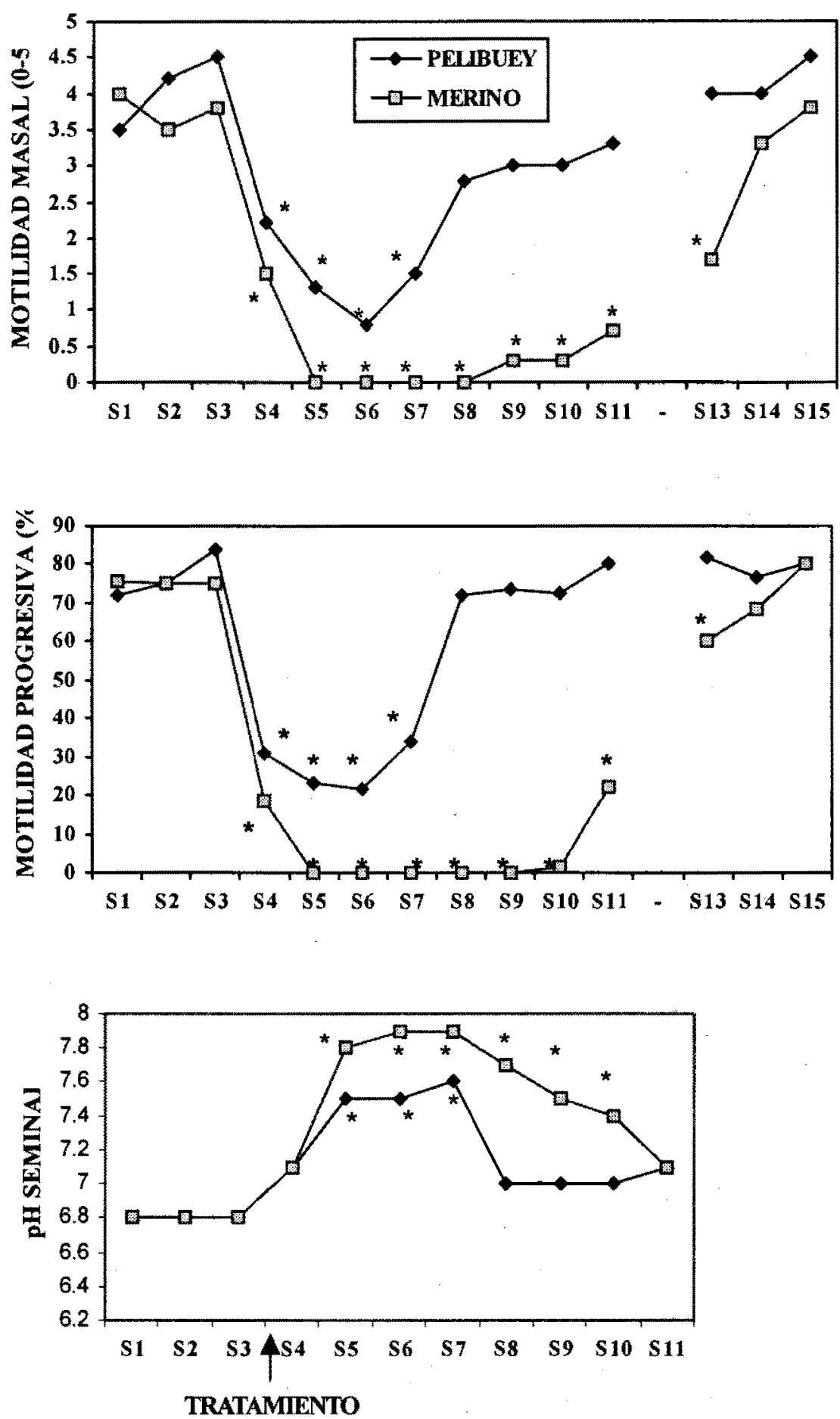

\section{SEMANAS DE COLECCIÓN DE SEMEN}

Figura 2. Efecto del incremento de la temperatura escrotal sobre la motilidad masal, motilidad progresiva de espermatozoides y pH seminal en ovinos Pelibuey y Merino Precoz durante las fases pre-experimental o control (S1 y S2), experimental (S3 a S11) y post-experimental (S13 a S15). Los * indican la diferencia significativa de los promedios de las semanas post-tratamiento respecto al promedio de las semanas control en cada raza. 


\section{Discusión}

La bolsa térmica aplicada a los testículos desarrolló una temperatura «in situ» de $37.1 \pm 0.9^{\circ} \mathrm{C}$ en ovinos Pelibuey y $38.2 \pm 0.7^{\circ} \mathrm{C}$ en ovinos Merino Precoz, por consiguiente la superficie externa del escroto expuesta a estas temperaturas resultó en alteraciones de las características seminales de $\mathrm{pH}$, concentración espermática, motilidad masal y progresiva, y frecuencia de espermatozoides muertos y anormales en ambas razas. Efectos similares han sido informados por otros autores, con similar o diferente metodología, en otras razas (Merino: Moule y Waites, 1963; Waites y Setchell, 1964; Branden y Mattner, 1970; Williamson, 1974 y Mieusset et al., 1992a; Southdown: Dutt y Hamm, 1957; Hampshire: Brooks y Ross, 1962), los resultados del presente estudio representa el primer reporte en ovinos Pelibuey y Merino Precoz con el uso de bolsas térmicas, sobretodo en un ambiente caluroso y húmedo en la costa (Lima) del país.

\section{Características seminales}

La magnitud del efecto del calor escrotal en las características seminales de las semanas posteriores al tratamiento dependió de la raza. El efecto del calor escrotal a las 2 y 3 semanas después del tratamiento en ambas razas, concuerda con otros reportes (Dutt y Hamm, 1957; Moule y Waites, 1963; Branden y Mattner, 1970; Williamson, 1974 y Mieusset et al., 1992a); sin embargo, el inicio del periodo de regeneración fue más tardío en Merino Precoz que en Pelibuey ( 77 vs. 46 días después del tratamiento), lo cual sugiere que el ovino Pelibuey tuvo ventajas fisiológicas para contrarrestar el efecto del calor en las características seminales e iniciar más temprano su regeneración; el resultado de esta característica es la interacción significativa de raza*semana obtenida en el presente estudio. Otros autores reportan un inicio de regeneración diferente (rango de 42 y 55 días después del tratamiento) (Dutt y Hamm, 1957; Moule y Waites, 1963; Waites y Setchell, 1964; Branden y Mattner, 1970); la diferencia puede ser debida a la intensidad de calor y al tiempo de exposición (Temperatura ambiental: $32^{\circ} \mathrm{C}$ por 7 días y $40.5^{\circ} \mathrm{C}$ por 2 horas, respectivamente) utilizados por los autores mencionados, ya que estas variables influyen marcadamente en la magnitud de la alteración de las características seminales (Setchell, 1998).

La mayor disminución en la concentración espermática en Merino Precoz $\left(0.04 \times 10^{6}\right)$ difiere con los reportes de Dutt y Hamm (1957) en ovinos Southdown (1.2x10 $0^{6}$ y Brooks y Ross (1962) en Hampshire $\left(1.4 \times 10^{6}\right)$; esta diferencia puede ser debida a que dichos autores utilizaron una temperatura ambiental que no superó $\operatorname{los} 32^{\circ} \mathrm{C}$, no obstante el efecto que obtuvieron fue el resultado de un mayor tiempo de exposición ( 7 y 60 días, respectivamente); sin embargo, similar resultado al reportado por estos autores fue obtenido en los carneros Pelibuey $\left(1.5 \times 10^{6}\right)$ a pesar de haber estado sometidos a un incremento de temperatura escrotal.

El incremento de los porcentajes de espermatozoides anormales y muertos dos semanas después del tratamiento en ambas razas concuerda con los resultados obtenidos por Moule y Waites (1963), Waite y Setchell (1964) y Branden y Mattner (1970). Sin embargo, hubo interacción de raza*semana, debido a una recuperación más temprana en ovinos Pelibuey que en Merino Precoz en la frecuencia de espermatozoides muertos y anormales. La falta de estudios con similar metodología y razas limitan la comparación de los resultados del presente estudio.

Las anormalidades más frecuentes halladas en el presente estudio coinciden con lo reportado por Dutt y Hamm (1957) y Williamson (1974), quienes también hallaron mayor porcentaje de cabezas desprendidas seguidas de colas dobladas. El tipo de anormalidades halladas, con excepción de cola doblada, pertenecen al grupo de anormalidades primarias, las cuales se producen por alteración del proceso espermatogénico (Sorensen, 1991); sin embargo, las colas 
dobladas cuando se hallan en alto porcentaje se debe a una grave alteración a nivel testicular (Derivaux, 1982).

En el presente estudio, la reducción en la motilidad masal debe ser consecuencia en parte de la disminución en la concentración espermática y a la mayor frecuencia de espermatozoides muertos, mientras que la reducción en la motilidad progresiva por la alteración de proteínas responsables de la motilidad, ya que aquellas que intervienen en la espermatogénesis son afectadas por el incremento del calor escrotal (McLaren et al., 1994). Evidencias similares sobre la motilidad espermática han sido reportadas en corderos Pelibuey (Lozano, 1982; citado por Valencia et al., 1990) y en otras razas de lana (Dutt y Hamm, 1957; Brooks y Ross, 1962; Moule y Waites, 1963; Howarth, 1969). Sin embargo, la magnitud del efecto fue menor y el periodo de regeneración más temprano en Pelibuey (interacción significativa de raza*semana). Carmenate et al. (1982) encontraron que de todas las características del plasma seminal en ovinos Corriedale y Pelibuey expuestos a la humedad relativa alta y temperatura ambiental de $28-30^{\circ} \mathrm{C}$, la presión osmótica fue la más sensible al efecto de la humedad relativa. El presente estudio se realizó en verano con alta humedad relativa ( $81 \%)$; por lo tanto también se esperaría un sinergismo interactivo entre temperatura (ambiental más escrotal) y humedad relativa, frente al cual el Merino Precoz fue el más susceptible.

El pH seminal en el presente estudio alcanzó valores superiores a 7.3 que es el máximo valor normal según McKenzie y Berliner (1937) (citados por Mann, 1964), y este efecto está relacionado a la alteración encontrada en la concentración y motilidad espérmatica, ya que el $\mathrm{pH}$ tiende a hacerse alcalino cuando disminuye la motilidad y el número de espermatozoides; esto debido a la menor formación de ácido láctico ante una pobre actividad motil (Mann, 1964).

El efecto simple de semana de colección tuvo tendencia a la significancia $(P<0.06)$ en volumen seminal, sin embargo al determinarse que esta diferencia ocurrió sólo en la semana $5(\mathrm{P}<0.06)$, la probabilidad de la tendencia disminuyó, lo cual sugiere que el efecto de semana fue muy débil o estuvo ausente. Esta tendencia podría deberse a alguna diferencia en la técnica de colección de semen y/o a la medición objetiva del volumen eyaculado. Dutt y Hamm (1957), Broks y Ross (1962), Moule y Waites (1963) y Howarth (1969) no hallaron alteración significativa del volumen seminal al exponer carneros a una temperatura ambiental y humedad relativa alta.

\section{Comportamiento Sexual}

La ausencia del efecto del tratamiento en el líbido sexual de los ovinos Pelibuey y Merino Precoz es similar al reporte de Godfrey et.al. (1998) en carneros de pelo St. Croix White y Barbados Blackbelly bajo temperatura tropical $\left(38.3 \pm 3^{\circ} \mathrm{C}\right.$ en sol y $33.1 \pm 3^{\circ} \mathrm{C}$ en sombra). Contrariamente, Lindsay (1969), encontró disminución de la actividad sexual de carneros Border Leicester, Dorset Horn y Merino dos semanas después de exponerlos a una temperatura ambiental de $43^{\circ} \mathrm{C}$; sin embargo, en este estudio los ovinos fueron inducidos a eyacular en la vagina artificial cada hora durante un periodo de 8 horas y es probable que este manejo estresante influyó en la disminución del líbido sexual.

\section{Susceptibilidad de los ovinos Pelibuey y Merino Precoz Alemán al Incremento de la Temperatura Escrotal}

En el presente estudio no hubo mayormente efecto significativo de raza y es probable que este efecto haya sido limitado por el número reducido de animales experimentales; sin embargo, la interacción significativa raza* ${ }^{*}$ semana encontrada en la alteración de las características seminales en estudio (excepto volumen), indica que el efecto del incremento de temperatura escrotal en cada semana después del tratamiento dependió de la respuesta de cada raza y muestra al ovino Merino Precoz como el más susceptible al mantener en forma prolongada la alteración de la función testicular. 
Esta diferente susceptibilidad ocurrió no obstante que en ambas razas el tratamiento fue loçal en los testículos y estuvieron expuestas a las mismas condiciones de temperatura y humedad relativa ambiental, además del hecho de adicionar fibra sintética alrededor de los testículos en los ovinos Pelibuey. La inferencia de estos efectos podría sugerir que el ovino Pelibuey tiene una mejor habilidad para compensar el efecto del incremento del calor escrotal. Fisiológicamente se conoce que los testículos poseen un mecanismo termorregulador, accionando por la regulación de la temperatura de la sangre arterial (caliente) y venosa (fría) (Setchell, 1987), complementado por la función del músculo cremáster, la túnica dartos, y las glándulas sudoríparas del escroto (Mieusset et al., 1992a).

- Otro factor que puede haber influido en la diferente susceptibilidad es la característica cárnica y el vellón crecido $( \pm 10$ meses de crecimiento) de los ovinos Merino Precoz a diferencia de la característica menos cárnica y la presencia de pelo en los Pelibuey.

Previo al tratamiento en el presente estudio, la $T^{\circ} \mathrm{R}$ fue mayor en Merino Precoz $\left(40.4^{\circ} \mathrm{C}\right)$ que en Pelibuey $\left(39.6^{\circ} \mathrm{C}\right)$ y esta diferencia se mantuvo durante el período de tratamiento $\left(39.9 \pm 0.6^{\circ}\right.$ vs. $39.2 \pm 0.6^{\circ} \mathrm{C}$ en Merino Precoz y Pelibuey, respectivamente). Similar diferencia ocurrió en la $\mathrm{T}^{\circ} \mathrm{E}\left(38.2 \pm 0.7^{\circ}\right.$ vs. $\left.37.1 \pm 0.9^{\circ} \mathrm{C}\right)$ y $1 \mathrm{a} \mathrm{T}^{\circ} \mathrm{P}\left(41.5 \pm 1.3^{\circ} v s\right.$. $39.8 \pm 1.0^{\circ} \mathrm{C}$ ). La correlación alta y significativa de la $\mathrm{T}^{\circ} \mathrm{E}$ con la $\mathrm{T}^{\circ} \mathrm{R}$ y $\mathrm{T}^{\circ} \mathrm{P}(\mathrm{r}=0.63,0.65$, respectivamente), indicaría fuertemente que el incremento de la $\mathrm{T}^{\circ} \mathrm{E}$ dependió, sobretodo, del incremento de la $T^{\circ} \mathrm{P}$ y explicaría en parte la susceptibilidad exhibida por el Merino Precoz.

El mecanismo fisiológico del mayor incremento de la $\mathrm{T}^{\circ} \mathrm{E}$ en el Merino precoz sería el efecto de la cubierta de lana y probablemente de su característica genética carnicera, en retener el calor del medio ambiente, limitando su disipación de la superficie corporal al exterior, con la consecuencia de un incremento en la temperatura corporal interna reflejado en la $T^{\circ} R$, y por ende en la sangre arterial, interfiriendo de esta manera con el mecanismo termo-regulador de los testículos. Esto sugiere que la habilidad del ovino Pelibuey para compensar el efecto del calor radicaría en la cobertura de su piel (pelo), lo que le permitiría disipar el calor más eficientemente en lugar de un eficiente mecanismo local termo-regulador de sus testículos.

Este análisis sugeriría que los ovinos Pelibuey y Merino Precoz en similar condición de cubierta corporal, tendrían similar respuesta al efecto del incremento de $\mathrm{T}^{\circ} \mathrm{E}$ sobre la función testicular. Dutt y Hamm (1957) encontraron que en grupos de carneros esquilados y no esquilados de la misma razas expuestos a un incremento de temperatura ambiental $\left(32^{\circ} \mathrm{C}\right.$ de temperatura y $65 \%$ de humedad relativa durante 7 días), los carneros esquilados no sufrieron alteración en la calidad seminal y en la $T^{\circ} \mathrm{R}$ a diferencia de los no esquilados. Sin embargo, no se descarta que la piel de los ovinos Pelibuey pueda poseer una actividad más eficiente de las glándulas sudoríparas, materia que merece ser investigada.

\section{Lichtira olmo}

1. Branden, A.W.H. y P.E. Mattner. 1970. The effects of scrotal heating in the ram on semen characteristics, fecundity, and embryonic mortality. Aust.J.agric.Res. 21:509-518.

2. Brooks,J.R. y C.V. Ross. 1962. Effect of ambient temperature and thiroxine therapy on semen quality of rams. J.Anim.Sci. 21:700-705.

3. Carmenate, C.; P. Gamicik y J.J. Hernández. 1982. Influencia de algunos factores climáticos sobre las características físicas y morfológicas del semen ovino. Rev. Cub. Reprod. Anim. (2):17-24.

4. Degen, A.A. y R.W. Benjamin. 1987. A note on increasing lamb producction 
of fat-tailed Awassi and German Mutton Merino sheep grazing in a semi-arid area. Prod.44:169-172.

5. Derivaux, J. 1982. Evaluación de la fertilidad masculina. En: Reproducción de los animales domésticos. 2da. Ed. P. 144168, Acribia, España.

6. Dutt,R.H. y P.T. Hamm. 1957. Effect of exposure to high environmental temperature and shearing on semen production of rams in winter. J.Anim.Sci. 16:328334.

7. Evans, G y W.M.C. Maxwell 1987. Salamon's artificial insemination of sheep and goats. p.85-106. Australia, Butterworths,

8. Godfrey,R.W.; J.R. Collins y M.L. Gray. 1998. Evaluation of sexual behavior of hair sheep rams in a tropical environment. J.Anim.Sci 76(3):714-717.

9. Hancock,J.L. 1952. The morfology of bull espermatozoa. J.Exp.Biol. 29:445.

10. Hochereau-de Reviers, M.T.; A. Locatelli; C. Perreau; C. Pissselet y B. P. Setchell. 1993. Effects of a single brief period of moderate heating of the testes of seminiferous tubules in hypophysectomized rams treated with pituitary extract. J.Reprod.Fert. 97:381-387.

11. Howarth,B. 1969. Fertility in the ram folowing exposure to elevated ambient temperature and humidity. J.Reprod.Fert. 19:179-183.

12. Lincoln, G.A. 1992. Photoperiod-pinealhypothalamic relay in sheep. Anim.Reprod.Sci. 28: 203-217.

13. Lindsay, D.R. 1969. Sexual activity and semen production of rams at high temperatures. J.Reprod.Fert. 18:1-8.

14. Mann,T. 1964. The biochemistry of semen and of the male reproductice tract. 493p. Methuen \& Co Ltd., Londres.

15. McLaten,T.T; P.M.D. Foster y R.M. Sharpe. 1994. Identification of stage changes in protein secretion by isolated seminiferous tubules from rats following exposure to short-term local testicular heating. J:Reprod.Fert. 102:293-300.

16. Mieusset,P; P. Quintana Casares; $G$ Sánchez Partida; S.F. Sowerbutts; J.L. Zupp y B.P. Setchell. 1992a. Effects of heating the testes and epididymides of rams by scrotal insulation on fertility and embryonic mortality in ewes inseminated with frozen semen. J.Reprod.Fert. 94:337-343.

17. Moule,GR. y GM.H. Waites. 1963. Seminal degeneration in the ram and its relation to the temperature of scrotum. J.Reprod.Fert. 5:433-446.

18. SAS Institute 1990. SAS/STAT User's Guide, Version 6, $4^{\text {th }}$ Ed. Vol. 1 and 2. SAS Institute Inc., NC, USA.

19. Setchell,B.P. 1987. Spermatogenesis and spermatozoa. En: Germ cells and fertilization. Reproduction in mammals. p:63-101. Ed. C.R. Austin y R.V. Short. Cambridge University Press, Gran Bretaña.

20. Setchell, B.P. 1998. The Parkes lecture. Heat and the testis. J.Reprod.Fert. 114:179-194.

21. Sorensen, A.M.Jr. 1991. Reproducción animal, principios y prácticas. McGrawHill, México.

22. Steel,R.GD. y Torrie,J.H. 1960. Principles and procedures of statistics. McGraw-Hill Book Company, Inc., USA.

23. Valencia,J; A. Gonzalez-Reyna y S.F. Lopez-Barbella. 1990. Hair sheep in Mexico and Venezuela: Reproduction in Pelibuey and West African sheep. In: Livestock reproduction in Latin America. P:299-320, Austria.

24. Waites, GM.H. y B.P. Setchell. 1964. Effect of local heating on blood flow and metabolism in the testis of conscious ram. J.Reprod.Fert. 8:339-349.

25. Williamson, $P$. 1974. The fine structure of ejaculated ram spermatozoa following scrotal heating. J.Reprod.Fert. 40:191-195. 